\title{
Local clouds: Ionization, temperatures, electron densities and interfaces, from GHRS and IMAPS spectra of $\epsilon$ Canis Majoris ${ }^{\star}$
}

\author{
C. Gry ${ }^{1,2}$ and E. B. Jenkins ${ }^{3}$ \\ 1 ISO Data Center, ESA Astrophysics Division, PO Box 50727, 28080 Madrid, Spain \\ ${ }^{2}$ Laboratoire d'Astronomie Spatiale, BP 8, 13376 Marseille Cedex 12, France \\ 3 Princeton University Observatory, Princeton, NJ 08544-1001, USA \\ e-mail: ebj@astro.princeton.edu
}

Received 20 September 2000 / Accepted 5 December 2000

\begin{abstract}
The composition and physical properties of several local clouds, including the Local Interstellar Cloud (LIC) in which the Sun is embedded, are derived from absorption features in the UV spectrum of the star $\epsilon$ CMa. We derive temperatures and densities for three components by combining our interpretations of the ionization balance of magnesium and the relative population of $\mathrm{C}_{\mathrm{II}}$ in an excited fine-structure level. We find that for the LIC $n_{\mathrm{e}}=0.12 \pm 0.05 \mathrm{~cm}^{-3}$ and $T=7000 \pm 1200 \mathrm{~K}$. We derive the ionization fractions of hydrogen and discuss the ionizing processes. In particular the hydrogen and helium ionizations in the LIC are compatible with photoionization by the local EUV radiation fields from the hot stars and the cloud interface with the hot gas. We confirm the detection of high ionization species: Si III is detected in all clouds and C IV in two of them, including the LIC, suggesting the presence of ionized interfaces around the local clouds.
\end{abstract}

Key words. ISM: solar neighbourhood - ISM: clouds - ISM: ionization - ISM: structure - stars: $\epsilon$ CMa - ultraviolet: ISM

\section{Introduction}

The star $\epsilon$ CMa (B2 II, $V=1.50, v \sin i=44 \mathrm{~km} \mathrm{~s}^{-1}$, $\ell=239.8, b=-11.3$ ) (Hoffleit \& Jaschek 1982) at a distance of $130 \mathrm{pc}$ (Perryman et al. 1997) is by far the brightest extreme ultraviolet source in the sky (Vallerga et al. 1993) and thus the main photoionization source in the Solar Neighborhood (Vallerga \& Welsh 1995; Vallerga 1998). This is principally due to the extraordinary emptiness of the line of sight to $\epsilon \mathrm{CMa}$, as we describe below. A beneficial aspect of the emptiness and hence the simplicity of the sight line is the opportunity for us to study with an unusual level of detail individual diffuse clouds in the local interstellar medium. This is the subject of this paper.

In particular, the star $\epsilon$ CMa provides an opportunity to observe the absorption spectrum of the Local Interstellar Cloud (LIC) surrounding our solar system. In most cases we obtained a good signal-to-noise ratio

Send offprint requests to: C. Gry, e-mail: cgry@iso.vilspa.esa.es

* Based on observations with the NASA/ESA Hubble Space Telescope, obtained at the Space Telescope Institute, which is operated by the Association of Universities for Research in Astronomy, Inc., under NASA contract NAS5-26555. for the absorption features, thereby allowing us to derive the chemical and physical properties within the clouds, including depletion, temperature, electron density and ionization.

The temperature of the LIC is usually derived from the width of absorption lines by profile fitting. Different measurements applied to various elements all point to a temperature of around $7000 \mathrm{~K}$, but with a relatively large error. The most precise measurements are provided by the observation of the HI L $\alpha$ line, because the low mass of hydrogen offers the best discrimination between thermal and turbulent line broadening when compared to the results from heavier elements. Using this method, Linsky et al. (1995) found $T=7000 \pm 900 \mathrm{~K}$ in the directions toward Capella and Procyon.

The electron density in the LIC has been determined in the lines of sight towards Sirius (Lallement et al. 1994) and $\epsilon$ CMa (Gry et al. 1995) using the ratio $N(\mathrm{Mg}$ II $) / N(\mathrm{Mg}$ I), towards $\delta$ Cas with the ratio $N(\mathrm{NaI}) / N$ (Ca II) (Lallement \& Ferlet 1997), and with the ratio $N\left(\mathrm{C}_{\text {II }}{ }^{*}\right) / N(\mathrm{C}$ II $)$ towards Capella (Wood \& Linsky 1997) and the white dwarf RE J1032+532 (Holberg et al. 1999). All methods give results that are around $0.1 \mathrm{~cm}^{-3}$, which is roughly the same order of magnitude as the neutral gas density. 
There are strong indications that the LIC and similar clouds in the Local Interstellar Medium (LISM) are partly ionized. The observed fractional ionization of hydrogen can be explained by the EUV radiation from white dwarf and other stars, in particular $\beta \mathrm{CMa}$ and $\epsilon \mathrm{CMa}$ (Vallerga 1998). Previous studies of the nearby line of sight toward $\beta$ CMa with the Goddard High Resolution Spectrograph (GHRS) on the Hubble Space Telescope (Dupin \& Gry 1998) and an independent facility, the Interstellar Medium Absorption Profile Spectrograph (IMAPS) (Jenkins et al. 2000) have shown that the two main clouds in that sightline present a very high ionization fraction of hydrogen, which could be explained by photoionization due to the combination of $\epsilon$ CMa and $\beta$ CMa if the clouds are located close enough to the stars.

An outstanding problem is that these and other stars do not produce enough photons with energies above $24.6 \mathrm{eV}$ to explain the high fractional ionization of helium in the LISM, as shown by the low value of $n(\mathrm{He} \mathrm{I}) / n(\mathrm{HI})$ (equal to 0.07 instead of the cosmic ratio of 0.1 , which indicates that helium is more ionized than hydrogen). The two main proposals to explain this phenomenon are i) the LISM is still recombining from a much more highly ionized state produced by a supernova-related energetic event in the recent past (Reynolds 1986; Frisch \& Slavin 1996; Lyu \& Bruhweiler 1996) and ii) the ionization of He is maintained by the diffuse EUV radiation emitted by conductive interfaces between the cloud edges and the hot gas filling the "Local Bubble" in which they are embedded (Slavin 1989; Slavin \& Frisch 1998).

In this paper we present GHRS spectra of $\epsilon \mathrm{CMa}$ over limited wavelength intervals between $1190 \AA$ and $1550 \AA$, ones that include the lines of N I, O I, C II and $\mathrm{C}_{\text {II }}{ }^{*}, \mathrm{~S}$ II, SiII, Si III, and $\mathrm{CIV}$ at a wavelength resolution $R \sim 100000$. We consider also a profile of $\mathrm{O}$ I at $1039 \AA$, recorded by IMAPS at $R \sim 60000$. We show how they shed light on the knowledge of characteristics of the nearby diffuse clouds such as temperatures, electron densities, abundances, and degree of ionization.

\section{Observations and data reduction}

Most of the observations presented here have been performed in late 1996 with the Ech-A grating of GHRS. All data were taken with the $00^{\prime \prime} 25$ Small Science Aperture $(\mathrm{SSA})$, the procedure FP-SPLIT $=4$ and a substepping of 4 samples per diode (for details of the instrumentation, see Soderblom et al. 1995). For data processing, we used the standard STSDAS procedures working in the IRAF environment. We assigned wavelengths from the standard calibration tables. An error of \pm 1 resolution element on the wavelength assignment is expected to arise from magnetic drifts.

The signal-to-noise ratio $(\mathrm{S} / \mathrm{N})$ for all Ech $\mathrm{A}$ data is about 200 when the flux is at the level of the stellar continuum. However there is a degradation of signal quality for features that appear in the bottoms of strong stellar lines. For most interstellar lines the $\mathrm{S} / \mathrm{N}$ ranges between

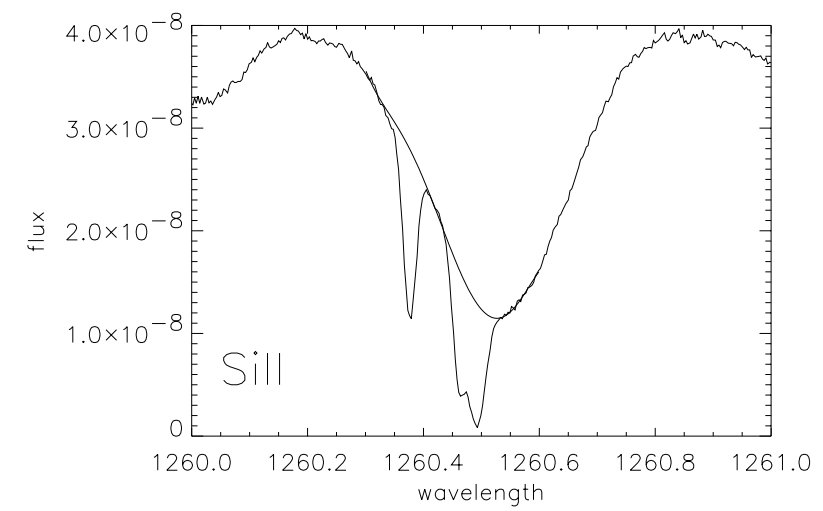

Fig. 1. Example of the continuum normalization for the Si II line at $1260.4 \AA$. The stellar line is fitted by a polynomial (shown here superimposed on the spectrum) over the velocity interval $\left[-30 \mathrm{~km} \mathrm{~s}^{-1}, 40 \mathrm{~km} \mathrm{~s}^{-1}\right]$

100 and 200, but it is 80 for $\mathrm{CII}$ and $\mathrm{CII}^{*}$ and 30 in the extreme case of Si III where the stellar line is the deepest.

Observations in the far-UV lines were carried out by IMAPS when it was operated on the ORFEUS-SPAS II mission that flew in late 1996 (Hurwitz et al. 1998). IMAPS is an objective-grating echelle spectrograph that was designed to record the spectra of bright, early-type stars over the wavelengths from $\sim 950 \AA$ to $\sim 1150 \AA$ with a high spectral resolution. For more details on the instrument see Jenkins et al. (1996).

The IMAPS spectra were extracted from the echelle spectral images using special procedures developed by one of us (EBJ) and his collaborators on the IMAPS investigation team. The $\mathrm{S} / \mathrm{N}$ obtained for the interstellar lines observed by IMAPS is of the order of 25 to 30 .

In both sets of spectra, there is an uncertainty related to the background correction for scattered light on the echelle format. For the GHRS Ech-A data, the error in the background correction is smaller than a few percent of the continuum and is only a concern for strong lines that almost reach the zero intensity level in their deepest points. In the case of a species for which several lines with different oscillator strengths are available, such as Si II, the uncertainty is eliminated by comparing the different profiles, which must have relative strengths that follow their oscillator strengths. For very strong lines like $\mathrm{H} \mathrm{I} \mathrm{Ly} \alpha$ and C II $1334 \AA$, the zero level is determined by the base of the saturated line. The situation for the O I $1302 \AA$ line is reported in Sect. 3.1.

Stellar lines were fitted with one low-order polynomial over a velocity range covering the interstellar components (Sect. 3), by considering the points bluewards of Component 4 up to $V=-30 \mathrm{~km} \mathrm{~s}^{-1}$ or $-40 \mathrm{~km} \mathrm{~s}^{-1}$, redwards of Component 1 up to $V=+40 \mathrm{~km} \mathrm{~s}^{-1}$, as well as the velocity range which is not affected by any interstellar absorption between Components 2 and 3. The spectra are then normalized to these stellar lines to create the interstellar profiles with a level continuum. An example of the stellar line fitting is illustrated in Fig. 1 for the Si II $1260 \AA$ line. For strong or moderately strong lines the uncertainty 
added to the column density estimates by this normalization process is small. However, for very faint lines ( $\mathrm{MgI}$, $\mathrm{C}_{\text {II* }}{ }^{*}, \mathrm{~S}$ II), it is a major source of error. This uncertainty was taken into account when we listed the column density results in Table 2 . The most difficult case is that for S II, where all three interstellar lines are very faint and appear on top of a steep slope of a stellar line. In this configuration there is a lot of freedom for the location of the synthetic stellar lines which can artificially enhance the interstellar line or, alternatively, make it disappear almost completely. We thus use several options for the SII absorption profiles defined by an envelope of $\pm 2 \sigma$ around the fitted continuum.

We derive the column densities by using the line fitting software "Owens" developed by Martin Lemoine. Each interstellar absorption component is represented by the convolution of a theoretical Voigt profile with the instrumental profile. The instrumental profile for the GHRS Echelle data was assumed to be a Gaussian with a FWHM of 0.92 diodes (Soderblom et al. 1995). An iterative procedure which minimizes the sum of the squared differences between model profiles and the data points allows us to determine the most likely column densities of the absorbing elements $N\left(\mathrm{~cm}^{-2}\right)$, the radial velocity of the cloud $\left(\mathrm{km} \mathrm{s}^{-1}\right)$ and the velocity dispersion (b-value) $\left(\mathrm{km} \mathrm{s}^{-1}\right)$ of each interstellar absorption component. The software also allows us to fit the lines from several elements simultaneously, which leads directly to a coherent solution for all species in terms of velocity, temperature and turbulent velocity. The wavelengths and $f$-values are listed in Table 1.

\section{Line of sight structure and column densities}

Previous high resolution spectra of UV absorption lines for the elements Fe II, MgII and Mg I obtained with the GHRS Ech B revealed a simple structure for the interstellar medium in the line of sight toward $\epsilon$ CMa (Gry et al. 1995): only three components were detected in all absorption lines, and their contributions amounted to an equivalent hydrogen column density of less than $10^{18} \mathrm{~cm}^{-2}$, a value that is by far the lowest amount ever observed in the Galactic disk for a line of sight as long as 130 pc. The low column densities offer the advantage that most of the UV absorption lines are unsaturated and can yield reliable column densities. Small, extra velocity components are detected in some of the strongest lines, as shown by Gry et al. (1995), however none of these exceeds an equivalent $\mathrm{H}$ column density of about $410^{16} \mathrm{~cm}^{-2}$.

The three principal components had heliocentric velocities of 17,10 and $-10 \mathrm{~km} \mathrm{~s}^{-1}$. In the previous papers, they were identified as Components 1, 2 and 3, respectively. Two of the components have been identified by Gry et al. (1995) as the two clouds detected in the line of sight to Sirius by Lallement et al. (1994) and described further by Hébrard et al. (1999). As Sirius is located at a distance of only $2.7 \mathrm{pc}$, these two components must be situated very close to the Sun.
Table 1. Observed atomic lines ${ }^{1}$

\begin{tabular}{|c|c|c|c|}
\hline & Element & wavelength $(\AA)$ & $f$-value \\
\hline IMAPS & O I & 1039.230 & $9.2010^{-3}$ \\
\hline \multirow[t]{21}{*}{ Ech A } & S III & 1190.203 & $2.3110^{-2}$ \\
\hline & Si II & 1190.416 & $2.9310^{-1}$ \\
\hline & Si II & 1193.290 & $5.8510^{-1}$ \\
\hline & $\mathrm{N}_{\mathrm{I}}$ & 1199.550 & $1.3010^{-1}$ \\
\hline & $\mathrm{N}_{\mathrm{I}}$ & 1200.223 & $8.6210^{-2}$ \\
\hline & $\mathrm{N}_{\mathrm{I}}$ & 1200.710 & $4.3010^{-2}$ \\
\hline & Si III & 1206.500 & $1.6710^{0}$ \\
\hline & D I & 1215.339 & $4.1710^{-1}$ \\
\hline & $\mathrm{H}$ I & 1215.670 & $4.1710^{-1}$ \\
\hline & S II & 1250.584 & $5.4310^{-3}$ \\
\hline & S II & 1253.811 & $1.0910^{-2}$ \\
\hline & S II & 1259.519 & $1.6610^{-2}$ \\
\hline & Si II & 1260.422 & $1.1810^{0}$ \\
\hline & O I & 1302.168 & $5.1910^{-2}$ \\
\hline & Si II & 1304.370 & $9.1710^{-2}$ \\
\hline & $\mathrm{C}_{\text {II }}$ & 1334.532 & $1.2810^{-1}$ \\
\hline & $\mathrm{C}_{\mathrm{II}}^{*}$ & 1335.708 & $1.1510^{-1}$ \\
\hline & Si IV & 1393.755 & $5.1410^{-1}$ \\
\hline & Si IV & 1402.770 & $2.5510^{-1}$ \\
\hline & $\mathrm{C}$ IV & 1548.195 & $1.9010^{-1}$ \\
\hline & C IV & 1550.770 & $9.4810^{-2}$ \\
\hline \multirow[t]{6}{*}{ Ech B } & Mg II & 2803.503 & $3.0610^{-1}$ \\
\hline & Mg II & 2796.352 & $6.1510^{-1}$ \\
\hline & $\operatorname{Mg} I$ & 2852.964 & $1.8310^{\circ}$ \\
\hline & Fe II & 2344.214 & $1.1410^{-1}$ \\
\hline & Fe II & 2382.765 & $3.2010^{-1}$ \\
\hline & Fe II & 2586.650 & $6.9110^{-2}$ \\
\hline
\end{tabular}

${ }^{1}$ Wavelengths and $f$-values are from a private communication by Morton, updating data from Morton (1991).

Component 1 is recognized as the Local Interstellar Cloud (LIC) in which the Sun is embedded, for which the motion has been characterized by Lallement \& Bertin (1992). Component 2, called the "Blue Cloud," is also in front of Sirius but is distinct from the LIC. Another component (Component 4) was detected on the blue side of Component 3 in the strongest lines at $-19 \mathrm{~km} \mathrm{~s}^{-1}$, while a fifth component was detected only in the line of Si III at $-65 \mathrm{~km} \mathrm{~s}^{-1}$, and confirmed in $\mathrm{H}$ i because it mimics a very strong D I absorption (too strong to be due to D I with a reasonable $D / H$ ratio), for its $\mathrm{H}$ I absorption turns out to coincide with the expected D I feature from Component 1 (see Sect. 3.3). Finally, a "Component 0" had been introduced in the red wing of Component 1 to improve the fit of some of the profiles, but our reanalysis with more complete data indicates that this component may not be real. Its introduction in the previous Ech B data analysis was probably a consequence of using a slightly distorted line spread function. We have chosen to omit the component in our more refined analysis.

We have performed the line fitting for Components 1 to 4 over all elements available in the Echelle A and Echelle B data, as well as for the O I line recorded by IMAPS. All lines of O I, Ni, Mg I, MgII, Fe II, Si II, Si III, C II, C II**, 

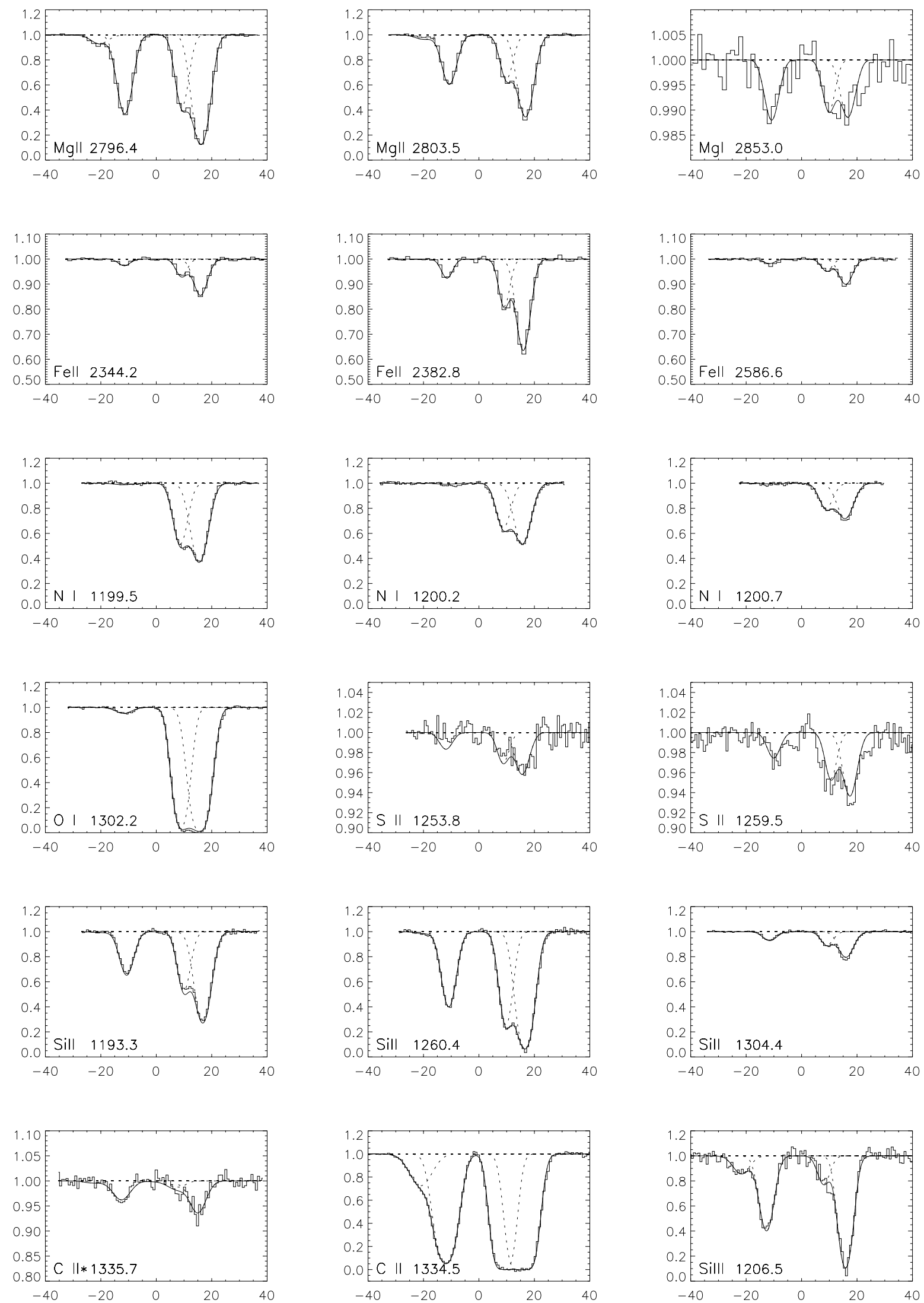

Fig. 2. Ech-B (first six plots) and new Ech-A spectra of $\epsilon$ CMa $(R \sim 100000)$, plotted in the heliocentric velocity scale $\left(\mathrm{km} \mathrm{s}^{-1}\right)$. The histogram-style tracings represent the observations, and the smooth solid lines indicate the fits to the interstellar absorption profiles. Dotted lines indicate the assumed stellar continua and the individual component contributions. The spectra have previously been divided out by a stellar absorption profile, as explained in Sect. 2 
Table 2. Column densities $\left(\mathrm{cm}^{-2}\right)$ of the five components detected toward $\epsilon$ CMa

\begin{tabular}{|c|c|c|c|c|c|}
\hline $\begin{array}{l}\text { Comp. } \\
V\end{array}$ & $\begin{array}{c}1 \\
17 \mathrm{~km} \mathrm{~s}^{-1}\end{array}$ & $\begin{array}{c}2 \\
10 \mathrm{~km} \mathrm{~s}^{-1}\end{array}$ & $\begin{array}{c}3 \\
-10 \mathrm{~km} \mathrm{~s}^{-1}\end{array}$ & $\begin{array}{c}4 \\
-19 \mathrm{~km} \mathrm{~s}^{-1}\end{array}$ & $\begin{array}{c}5 \\
-65 \mathrm{kms}^{-1}\end{array}$ \\
\hline Fe II & $1.35 \pm 0.0510^{12}$ & $5.2 \pm 0.510^{11}$ & $1.99 \pm 0.110^{11}$ & - & - \\
\hline Mg II & $3.1 \pm 0.110^{12}$ & $1.05 \pm 0.0510^{12}$ & $1.39 \pm 0.0510^{12}$ & $9.0 \pm 410^{10}$ & - \\
\hline $\mathrm{Mg} \mathrm{I}$ & $7 \pm 210^{9}$ & $5 \pm 210^{9}$ & $7 \pm 310^{9}$ & - & - \\
\hline Si II & $4.52 \pm 0.210^{12}$ & $1.85 \pm 0.110^{12}$ & $1.38 \pm 0.0510^{12}$ & $2.9 \pm 210^{10}$ & - \\
\hline Si III & $2.3 \pm 0.210^{12}$ & $2.0 \pm 1.110^{11}$ & $1.0 \pm 0.110^{12}$ & $2.2 \pm 0.510^{11}$ & $2.1 \pm 0.310^{11}$ \\
\hline $\mathrm{C}_{\text {II }}{ }^{a}$ & $(1.4-2.1) 10^{14}$ & $(0.7-1.3) 10^{14}$ & $5.21 \pm 0.210^{13}$ & $7.3 \pm 210^{12}$ & $2.3 \pm 0.310^{12}$ \\
\hline $\mathrm{C}_{\mathrm{II}}{ }^{*}$ & $1.3 \pm 0.210^{12}$ & $2.0 \pm 1.110^{11}$ & $7.4 \pm 1.010^{11}$ & - & - \\
\hline O I & $1.4_{-0.2}^{+0.5} 10^{14}$ & $1.2 \pm 0.310^{14}$ & $1.93 \pm 0.210^{12}$ & - & - \\
\hline N I & $1.70 \pm 0.0510^{13}$ & $9.8 \pm 0.510^{12}$ & $<2.210^{11}$ & - & - \\
\hline S II & $8.6 \pm 2.110^{12}$ & $4.9 \pm 1.510^{12}$ & $3.5 \pm 0.910^{12}$ & - & - \\
\hline $\mathrm{CIV}$ & $1.2 \pm 0.310^{12}$ & - & $3.7 \pm 0.410^{12}$ & - & - \\
\hline S III & $<310^{12}$ & $<310^{12}$ & $<310^{12}$ & - & - \\
\hline Si IV & $<210^{11}$ & $<210^{11}$ & $<210^{11}$ & - & - \\
\hline $\mathrm{N} v$ & $<2.510^{11}$ & $<2.510^{11}$ & $<2.510^{11}$ & - & - \\
\hline
\end{tabular}

${ }^{a}$ The upper limits for Components 1 and 2 are determined from $N(\mathrm{~S}$ II) as explained in Sect. 3.2.

and S II were fitted simultaneously with a unique line-ofsight velocity structure and a unique column density that was consistent with different lines of the same species. As the absolute wavelength calibration had a precision of one resolution element (about $3 \mathrm{~km} \mathrm{~s}^{-1}$ ), an individual velocity shift is allowed for each data set, whereas the relative velocity of the components had to be the same for all lines. The resulting velocity shifts for all elements have a dispersion of $\pm 0.94 \mathrm{~km} \mathrm{~s}^{-1}$, in agreement with the precision of the wavelength calibration. The derived velocity for Component 1 is $16.15 \pm 1.5 \mathrm{~km} \mathrm{~s}^{-1}$, in agreement with its identification as the Local Interstellar Cloud. The velocity shifts of the other components relative to Component 1 are known with more precision: $-6.92 \pm 0.03 \mathrm{~km} \mathrm{~s}^{-1}$ for Component 2, $-27.50 \pm 0.04 \mathrm{~km} \mathrm{~s}^{-1}$ for Component 3, and $-36.4 \pm 0.3 \mathrm{~km} \mathrm{~s}^{-1}$ for Component 4 .

The comparison of the synthetic profiles with the data suggested that the velocity shift between Component 3 and Component 1 could be slightly different for Si III from what we found for the other elements. We thus decided to decouple the Si III velocity from the determinations for other species, and we found that indeed the velocity shift between Component 3 and Component 1 for Si III is $-28.48 \mathrm{~km} \mathrm{~s}^{-1}$, i.e., $1 \mathrm{~km} \mathrm{~s}^{-1}$ more than for the other elements. This velocity shift however is only one-third of a resolution element and must be compared to the velocity dispersion of material in the clouds which is more than a factor of three higher.

When we derived $b$-values that had the best fit to the data, we imposed the requirement that various elements should have common values for the turbulent and thermal doppler contributions within each velocity component. Differences in the outcomes for $b$ across different elements were therefore governed only by variations
Table 3. $b$-values (velocity dispersion in $\mathrm{km} \mathrm{s}^{-1}$ ) derived from the fits. They are given for the elements carbon and iron, which represent the two extremes of atomic mass. The $b$-values for the other elements are intermediate between the two

\begin{tabular}{lcccc}
\hline Comp. & 1 & 2 & 3 & 4 \\
\hline C II & $3.9 \pm 0.3$ & $3.2 \pm 0.13$ & $4.0 \pm 0.1$ & $5.5 \pm 0.1$ \\
Fe II & $3.0 \pm 0.15$ & $2.12 \pm 0.17$ & $2.44 \pm 0.02$ & $4.4 \pm 0.4$ \\
\hline
\end{tabular}

in atomic mass. Table 3 shows the $b$-values for two elements that represent the two extremes in mass: carbon and iron.

The derived column densities for Components 1 to 4 are listed in Table 2, and the fits of the lines are presented in Fig. 2. Specific considerations for a few elements are discussed individually below.

\subsection{Ol column density}

Guidance on the correct zero level of the O I line at $1302 \AA$ was available from the observed zero level of the nearby C II line at $1334 \AA$ which is known to be heavily saturated. The presence of clearly visible component structures at the bottom of the line indicates that the profile remains above zero or, at most, it hits zero over a range of just a few points, in accord with the expected noise level. However an uncertainty on the exact zero level location still remains, and this is manifested as an uncertainty in the column densities for Components 1 and 2. It is thus useful to check the results using the weaker line of O I at $1039 \AA$, which is available in the IMAPS data.

The interstellar O I feature observed with IMAPS is unfortunately contaminated by telluric $\mathrm{O}$ I absorption, as 

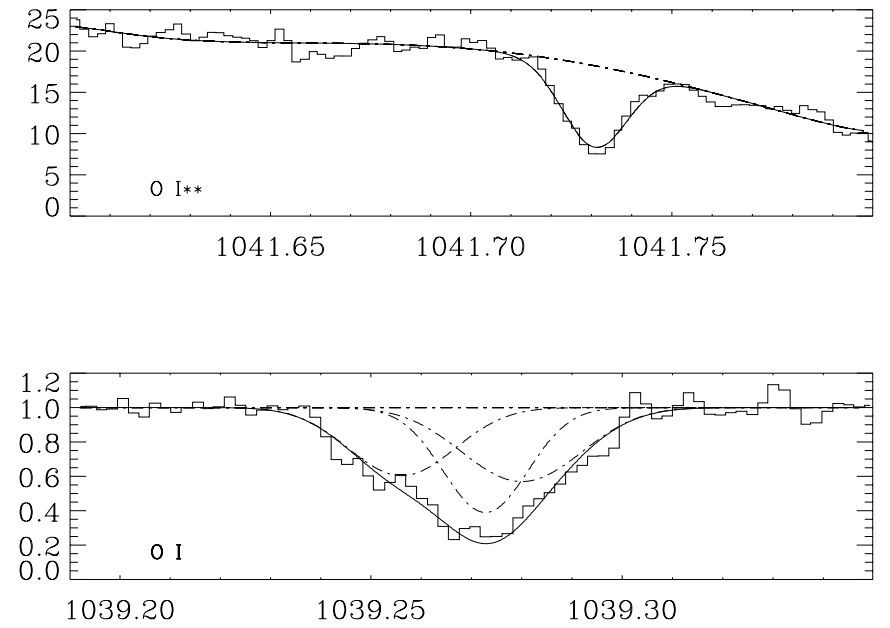

Fig. 3. IMAPS spectra of $\epsilon$ CMa $(R \sim 60000)$ for the line of O I at $1039 \AA$ and O I** at $1041 \AA$. The histogram-style tracings represent the observations and the smooth solid lines the fits to the interstellar and telluric absorption profiles. Also shown in dash-dotted lines are the assumed stellar continuum and the individual component contributions. In the $\mathrm{O}^{* *}$ line, only the telluric component contributes to the profile while the $\mathrm{O}$ I line is a blend of the interstellar components 1 and 2 and the telluric line (the telluric component is the central one)

revealed by the presence of a strong, narrow $\mathrm{O}^{* * *}$ absorption line at $1041 \AA$. However we can estimate the expected telluric contribution for O I. In the Earth's upper atmosphere, the fine-structure levels should be populated in accord with their statistical weights. With this in mind, we performed a fit of the far-UV O I and $\mathrm{O}_{\mathrm{I}}^{* *}$ lines together with the other species (leaving out the O I $1302 \AA$ line), but with the addition of a telluric component satisfying the constraint that $N(\mathrm{OI})=5 \times N\left(\mathrm{OI}^{* *}\right)$. The resulting fits are illustrated in Fig. 3. They give results for the $\mathrm{O} I$ column densities of Components 1 and 2 that agree with the range of values derived from the O I $1302 \AA$ line alone, giving an assurance that the background assignment for that line was not erroneous. Table 2 lists the O I column densities which are consistent with both $\mathrm{O}$ I lines.

\subsection{CII column density}

There are substantial uncertainties with the CII column density determinations for Components 1 and 2 because the line is so strong. Over the velocity range covered by Component 1 , the profile shows nearly zero intensity - a situation that is consistent with arbitrarily high column densities. Therefore, while some lower limit for $N(\mathrm{C}$ II $)$ could be gathered from the profile fitting, we derived upper limits from the upper limits for $N(\mathrm{~S}$ II $)$ multiplied by the cosmic abundance ratio, $(N(\mathrm{C} \text { II }) / N(\mathrm{~S} \text { II }))_{\text {cosmic }}=20$ (Anders \& Grevesse 1989). In effect, S II and CII are both the dominant ionization states in diffuse neutral gas, and even if these two elements are in a partially ionized medium, they should have about the same fraction of atoms elevated to higher stages of ionization (Jenkins et al. 2000). While this may be true, we also know that carbon is usually more depleted than sulfur in the ISM, implying that $N(\mathrm{C}$ II $) / N(\mathrm{~S}$ II $)$ is probably lower than the cosmic abundance ratio. The upper limits for $N(\mathrm{C}$ II $)$ listed in Table 2 for Components 1 and 2 reflect these considerations. For Component 3, the line is not as saturated, and we derived both the lower and the upper limits from the line fitting, and we note that the upper limit we measure is very close to the value we would estimate from S II.

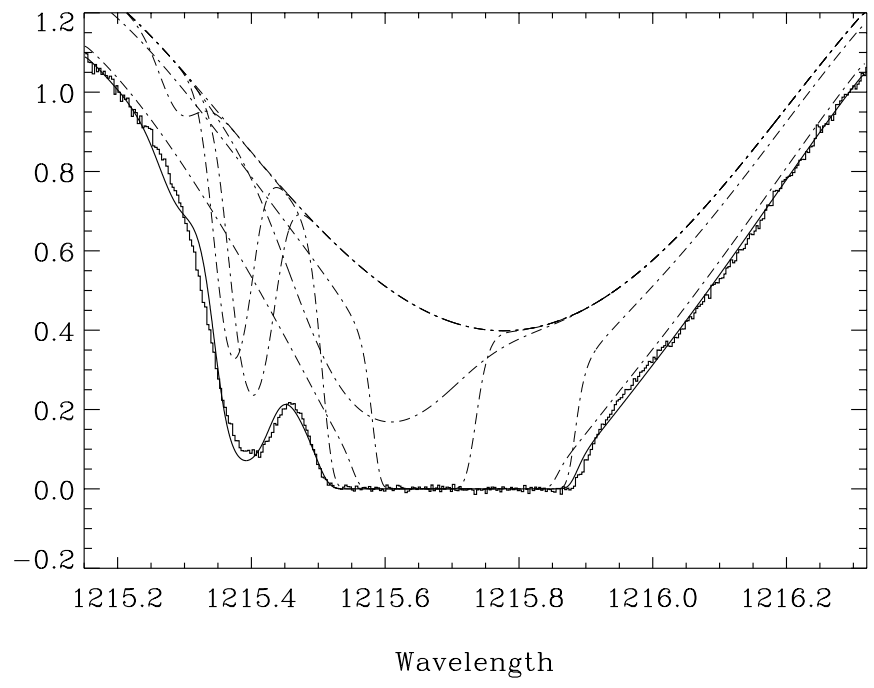

Fig. 4. The four interstellar components of H I and D I fitted on the stellar Lyman $\alpha$ profile of $\epsilon \mathrm{CMa}$, represented by a fourth order polynomial, the coefficients of which are also free parameters in the fit. This presentation illustrates the complex superposition of profiles. It is impossible to find a unique solution for the H I and D I column densities, partly because of the impossibility to define the stellar continuum independently

\subsection{HI and DI Lyman $\alpha$ profiles}

In principle, the low column density of neutral matter present in the line of sight toward $\epsilon \mathrm{CMa}$ is favorable for studying $D / H$ because the bottom of the $\mathrm{H}$ l line is not wide enough to bury the deuterium line. Indeed, as seen in Fig. 4, the D I feature is clearly seen in the wing of the H I profile. Unfortunately, as already noted by Gry et al. (1995) with the G160M data, it is very difficult to derive $\mathrm{HI}$ and DI column densities from the Lyman $\alpha$ line because

i) the H I stellar line is narrow compared to the interstellar profile and is thus very difficult to define with any accuracy;

ii) the D I feature is probably blended with an H I absorption from the small component at $-65 \mathrm{~km} \mathrm{~s}^{-1}$. Although this component is very weak and probably mostly ionized, for it is detected only in Si III and C II, a neutral hydrogen column density of only $10^{13} \mathrm{~cm}^{-2}$ could dominate over a deuterium feature if $D / H \sim 210^{-5}$;

iii) since the mass of deuterium is low, its $b$-value is high 
and the absorption features from the different components are not resolved very well, unlike the cases for the other elements. This makes it impossible to distinguish the contribution of each component to the D I profile, which might otherwise allow us to separate a blend of three or four D I components from the one $\mathrm{H}$ i high velocity component.

We plot in Fig. 4 some representative fits to the Lyman $\alpha$ profile simply to illustrate the complexity arising from the overlapping individual absorbers. In this example the Lyman $\alpha$ profile has been fitted using information from the other elements, without any additional constraints. This solution is not acceptable because it implies an $\mathrm{HI}$ column density for Component 2 which is incompatible with the limit set by O I or S II. We have checked nevertheless that the H I column densities derived from O I in Sect. 4.1 produce a synthetic Lyman $\alpha$ profile which is compatible with the observed profile.

\subsection{CIV profiles}

Some absorption features can be seen on the EchA spectra at the bottom of the two C IV stellar lines (Fig. 5). They were already apparent at lower resolution in the G160M data (Gry et al. 1995). If we fit a stellar continuum outside the range where the contributions from Components 1 and 3 are expected, the normalized spectra show two components with a velocity separation which is compatible with the separation between Components 1 and 3 with column densities of $1.2 \pm 0.310^{12}$ and $3.7 \pm 0.410^{12} \mathrm{~cm}^{-2}$, respectively.

There is a shift of about $-5 \mathrm{~km} \mathrm{~s}^{-1}$ in the absolute velocity of the components, compared to the velocities of these components measured for the other species. This shift is a bit too high to be attributed to an uncertainty in the wavelength calibration, which should be less than $3 \mathrm{~km} \mathrm{~s}^{-1}$ (this has been verified with the other elements). However, velocity discrepancies between highly ionized species and lower ionization stages are not unusual; see, e.g., Sembach et al. (1994).

We have also performed an evaluation of the $\mathrm{C}$ IV lines with a fit to the stellar and interstellar lines simultaneously over a velocity interval that includes the region covered by Components 1 to 3 and without specifying the velocity of the interstellar components. This approach eliminates the possible bias that could arise from the a priori choice of the location of the interstellar features when defining the stellar continuum. The result of the fit was virtually the same as that found with our standard way of deriving a normalized spectrum. Once again, a velocity separation equivalent to that between Components 1 and 3 was found. This separate exercise reinforces our proposal that the $\mathrm{CIV}$ lines are real and not simply a consequence of our incorporating information from other elements in lower stages of ionization.
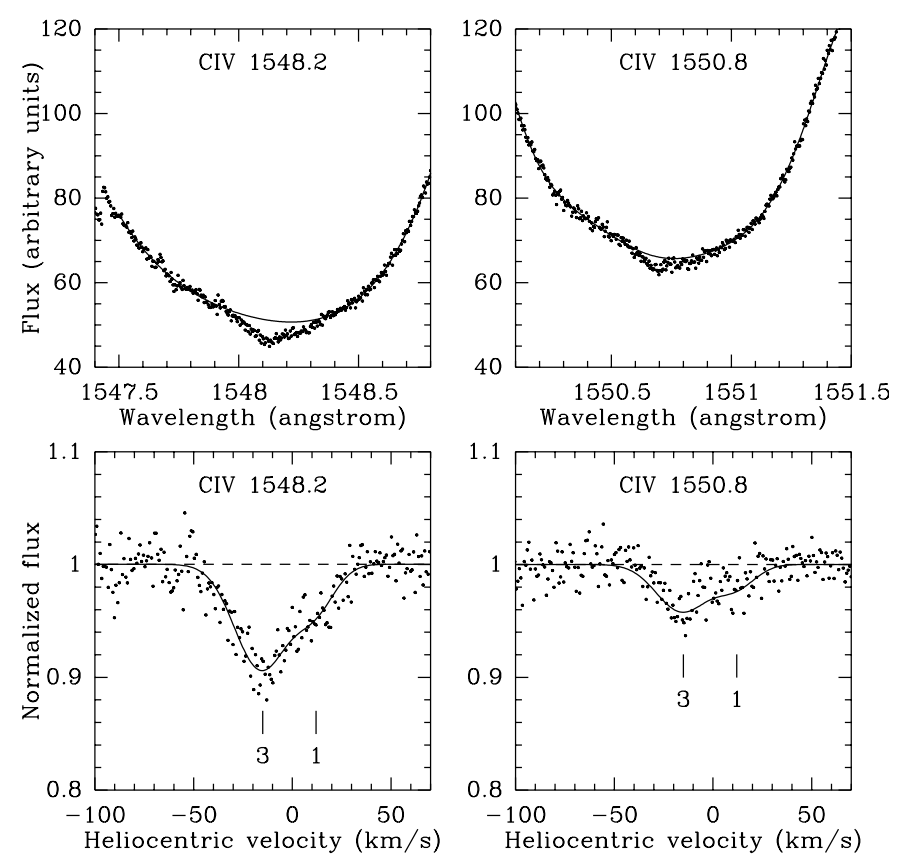

Fig. 5. The CIV profiles of $\epsilon \mathrm{CMa}$ at $1548.2 \AA$ (left) and $1550.8 \AA$ (right). This figure shows the fit of the stellar line by a fourth order polynomial (top), followed by the residuals arising from interstellar absorption with two components (bottom). A fit (solid line) indicates the existence of two components with a velocity separation equal to that of Components 1 and 3 derived from the other elements. However the absolute velocity of $\mathrm{C} I \mathrm{IV}$ is shifted by $-5 \mathrm{~km} \mathrm{~s}^{-1}$ compared to the velocities derived from the other species. The temperatures implied by the fits are equivalent to a kinetic temperature within the range 100000 to $200000 \mathrm{~K}$. The column densities are given in Table 2

\subsection{Other high ionization species}

Absorption features from the OVI doublet have been looked for in the IMAPS spectrum but they are not visible. None of the other high ionization species Si IV, S III and $\mathrm{N} v$ have been detected in the GHRS spectrum.

\section{Neutral and total gas column densities}

\subsection{Neutral gas column densities}

The ionization fractions of oxygen and nitrogen are coupled to that of hydrogen via resonant charge exchange reactions. In particular, the rate coefficient for charge exchange of $\mathrm{O}$ II with $\mathrm{H}$ I is exceptionally strong, $\sim 10^{-9} \mathrm{~cm}^{3} \mathrm{~s}^{-1}$ (Field \& Steigman 1971), and O I is therefore a very good tracer of $\mathrm{HI}$. We can thus derive the column densities of neutral hydrogen in the components from their respective $\mathrm{O}$ I column densities through the relation $N_{\mathrm{OI}}(\mathrm{HI})=N(\mathrm{O} \mathrm{I}) /(\mathrm{O} / \mathrm{H})_{\text {ISM }}$, where $(\mathrm{O} / \mathrm{H})_{\text {ISM }}$ is the abundance of oxygen relative to hydrogen in the interstellar medium. If we adopt the value derived by Meyer et al. $(1998),(\mathrm{O} / \mathrm{H})_{\mathrm{ISM}}=3 \cdot 1610^{-4}$, we find that $N_{\mathrm{OI}}(\mathrm{HI})=4.4_{-0.6}^{+1.6} 10^{17} \mathrm{~cm}^{-2}$ for Component 1 , $N_{\mathrm{OI}}(\mathrm{H} \mathrm{I})=3.8 \pm 0.910^{17} \mathrm{~cm}^{-2}$ for Component 2 and $N_{\mathrm{OI}}(\mathrm{H} \mathrm{I})=6.1 \pm 0.610^{15} \mathrm{~cm}^{-2}$ for Component 3 . 
With these numbers, we arrive at a total H I column density in the range $710^{17}$ to $1.110^{18} \mathrm{~cm}^{-2}$, which is compatible with that derived from the absorption of the extreme ultraviolet flux from the star based on EUVE spectra (Vallerga et al. 1993; Cassinelli et al. 1995).

If we adopt a different tactic by assuming that the diffuse clouds in the local ISM are undepleted and the oxygen abundance is equal to the abundance in B stars, $(\mathrm{O} / \mathrm{H})_{*}=4.6810^{-4}$, then the $\mathrm{H}$ I column density is lower, ranging from $4.810^{17}$ to $7.210^{17} \mathrm{~cm}^{-2}$ for the whole line of sight, with

$N_{\mathrm{OI}}(\mathrm{H} \mathrm{I})=3.0_{-0.4}^{+1.1} 10^{17} \mathrm{~cm}^{-2}$ for Component 1 , $N_{\mathrm{OI}}(\mathrm{H} \mathrm{I})=2.6 \pm 0.610^{17} \mathrm{~cm}^{-2}$ for Component 2 and $N_{\mathrm{OI}}(\mathrm{H} \mathrm{I})=4.1 \pm 0.410^{15} \mathrm{~cm}^{-2}$ for Component 3.

We can also derive the $\mathrm{H}$ I column densities implied by the N I column densities. If we again use the abundance of nitrogen in the interstellar medium derived by Meyer et al. (1997), $(\mathrm{N} / \mathrm{H})_{\mathrm{ISM}}=7.510^{-5}$, we find

$N_{\mathrm{N}_{\mathrm{I}}}(\mathrm{HI})=2.1 \pm 0.210^{17} \mathrm{~cm}^{-2}$ for Component 1 ,

$N_{\mathrm{N}_{\mathrm{I}}}(\mathrm{HI})=1.3 \pm 0.110^{17} \mathrm{~cm}^{-2}$ for Component 2 , and

$N_{\mathrm{NI}_{\mathrm{I}}}(\mathrm{HI}) \leq 2.910^{15} \mathrm{~cm}^{-2}$ for Component 3 .

We note that in all three components, the neutral column density derived from $\mathrm{N}_{\mathrm{I}}$ is significantly lower than that derived from O I. This N I deficiency in the local ISM has already been noticed by Jenkins et al. (2000) from far-UV spectra of white dwarf stars observed with FUSE. They have shown that the Ni deficiency, as that of Ar I, favors the existence of a source of ionizing photons with $E \geq$ $24.6 \mathrm{eV}$ in the local ISM to explain the He I ionization. For the subsequent discussions, we will adopt for $N(\mathrm{HI})$ the ranges derived from $N(\mathrm{O} \mathrm{I})$, keeping both options for the oxygen depletion.

\subsection{Total gas column densities}

S II is traditionally used as an indicator of the total (i.e. neutral plus ionized) gas column density in the interstellar medium because i) sulphur has little or no depletion onto dust grains (see, e.g., Savage \& Sembach 1996; Fitzpatrick \& Spitzer 1997) and ii) the ionization potential of S II (23 $\mathrm{eV}$ ) is high and therefore $\mathrm{SI}$ is often assumed to be the dominant ionization stage both in HI and HII regions. If we trust that assumptions i) and ii) are valid, we can derive the total column densities for Components 1, 2 and 3 from the expression: $N_{\mathrm{S} \text { II }}\left(\mathrm{H}_{\text {tot }}\right)=N(\mathrm{~S} \mathrm{II}) /(\mathrm{S} / \mathrm{H})_{\text {cosmic }}$, where $(\mathrm{S} / \mathrm{H})_{\text {cosmic }}$ is the cosmic abundance ratio taken from Anders \& Grevesse (1989): $(\mathrm{S} / \mathrm{H})_{\text {cosmic }}=1.8410^{-5}$. In this context, we obtain

$N_{\text {SII }}\left(\mathrm{H}_{\text {tot }}\right)=4.7 \pm 1.110^{17} \mathrm{~cm}^{-2}$ for Component 1 , $N_{\text {SII }}\left(\mathrm{H}_{\text {tot }}\right)=2.7 \pm 0.810^{17} \mathrm{~cm}^{-2}$ for Component 2, and $N_{\mathrm{S} \text { II }}\left(\mathrm{H}_{\text {tot }}\right)=2.0 \pm 0.510^{17} \mathrm{~cm}^{-2}$ for Component 3 .

\section{Electron density and temperature}

We follow the method of Jenkins et al. (2000) to derive both the temperature and the electron density in a component by combining the information from two ratios which depend in different manners on these two physical parameters.

The relative populations of the fine-structure levels of CII are governed by the balance between collisions and radiative de-excitation. In the diffuse warm medium, the collisions are dominated by electrons. The condition for equilibrium

$n(\mathrm{e}) \gamma_{1,2} n(\mathrm{C}$ II $)=\left[n(\mathrm{e}) \gamma_{2,1}+A_{2,1}\right] n(\mathrm{C}$ II $*)$

leads to an equation for the electron density

$n(\mathrm{e})=\frac{g_{2} A_{2,1} T^{0.5}\left[\frac{n(\mathrm{C} \text { II } *)}{n(\mathrm{C} I \mathrm{II})}\right]}{8.6310^{-6} \Omega_{1,2}\left\{\left(\frac{g_{2}}{g_{1}}\right) \exp \left(\frac{-E_{1,2}}{k T}\right)-\left[\frac{n(\mathrm{C} \text { II } *)}{n(\mathrm{C} \text { II })}\right]\right\}}(2)$

where the radiative decay probability for the upper level is $A_{2,1}=2.2910^{-6} \mathrm{~s}^{-1}$ (Nussbaumer \& Storey 1981), and the collision strength $\Omega_{1,2}=2.81$ (Hayes \& Nussbaumer 1984). Equation (2) thus gives a curve of $n(\mathrm{e})$ versus $T$ for any value of the ratio $n\left(\mathrm{CII}^{*}\right) / n(\mathrm{C}$ II $)$.

To see the conditions from a different perspective, we make use of the equation for the equilibrium between the two lowest ionization levels of magnesium which is given by

$$
\begin{array}{r}
{\left[\Gamma(\mathrm{Mg} \mathrm{I})+C(\mathrm{Mg} \mathrm{II}) n\left(\mathrm{H}^{+}\right)\right] n(\mathrm{Mg} \mathrm{I})=} \\
\alpha(\mathrm{Mg} \mathrm{I}) n(\mathrm{e}) n(\mathrm{Mg} \mathrm{II}) .
\end{array}
$$

For the charge exchange rate $C(\mathrm{Mg}$ II $)$ that applies to the reaction $\mathrm{MgI}+\mathrm{H}^{+} \rightarrow \mathrm{Mg} \mathrm{II}+\mathrm{H}$, we used the analytical approximation $C(\mathrm{Mg} \mathrm{II})=1.7410^{-9} \exp \left(-2.21 \quad 10^{4} / T\right)$ derived by Allan et al. (1988). We calculate $\Gamma(\mathrm{Mg} \mathrm{I})=$ $6.110^{-11} \mathrm{~s}^{-1}$ at the position of the Sun (for details, see Jenkins et al. 2000). For $\alpha(\mathrm{Mg} \mathrm{I})$ we used the radiative and dielectronic recombination rates given by Shull \& van Steenberg (1982), supplemented by the additional contributions from low-lying resonance states computed by Nussbaumer \& Storey (1986). As with Eq. (2), an application of Eq. (3) also provides a curve giving $n(\mathrm{e})$ versus $T$, but this time for any value of the ratio $N(\mathrm{Mg} \mathrm{II}) / N(\mathrm{Mg} \mathrm{I})$.

In Fig. 6, we plot for each component the curve $n(\mathrm{e})$ versus $T$ of Eq. (2) for the maximum and the minimum values of the ratios $n(\mathrm{CII}) / n(\mathrm{C}$ II $)$ derived from the observations, together with the two curves $n(\mathrm{e})$ versus $T$ given by Eq. (3) for the two extremes for the ratio $N(\mathrm{Mg}$ II $) / N(\mathrm{Mg}$ I). The shaded area bounded by the two sets of curves defines the possible values of $n(\mathrm{e})$ and $T$ that are consistent with both methods for each component.

We derive $5700<T<8200 \mathrm{~K}$ with $0.08<n_{\mathrm{e}}<$ $0.17 \mathrm{~cm}^{-3}$ for the LIC (Component 1), $8200<T<$ $30000 \mathrm{~K}$ with $0.016<n_{\mathrm{e}}<0.088 \mathrm{~cm}^{-3}$ for Component 2 , and $6000<T<8400 \mathrm{~K}$ with $0.18<n_{\mathrm{e}}<0.28 \mathrm{~cm}^{-3}$ for Component 3 .

Note that our results confirm the temperature of $7000 \mathrm{~K}$ commonly found for the LIC in most studies, and our electron density of $n_{\mathrm{e}}=0.12 \pm 0.05 \mathrm{~cm}^{-3}$ confirms the ranges found independently in the LIC in other sightlines by Wood \& Linsky (1997) and Holberg et al. (1999). From our results, the LIC and Component 3 have similar temperatures while Component 2 could be warmer. 

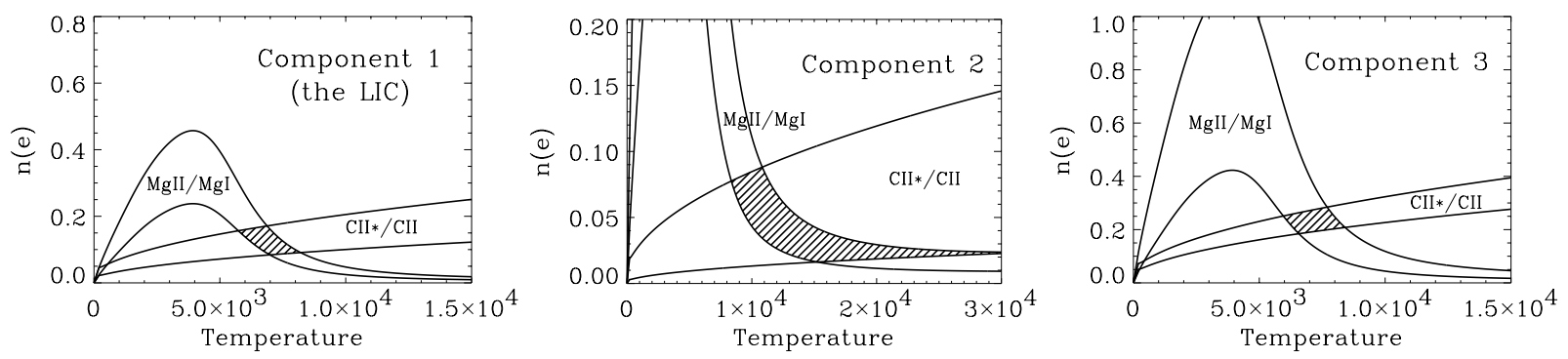

Fig. 6. Curves of the electron density $n(\mathrm{e})$ versus temperature $T$ governed by Eqs. (2) and (3), for the permitted values of the ratios $n\left(\mathrm{CII}^{*}\right) / n(\mathrm{CII})$ and $N(\mathrm{Mg} \mathrm{II}) / N(\mathrm{Mg} \mathrm{I})$. For each of the three components the shaded area where the two sets of curves intersect determines the possible values for $n(\mathrm{e})$ and $T$

Table 4. Derived properties of the three main components

\begin{tabular}{lccc}
\hline Comp. & $1(\mathrm{LIC})$ & 2 & 3 \\
\hline$T(\mathrm{~K})$ & $5700-8200$ & $8200-30000$ & $6000-8400$ \\
$n_{\mathrm{e}}\left(\mathrm{cm}^{-3}\right)$ & $0.08-0.17$ & $0.016-0.088$ & $0.18-0.28$ \\
$n_{\mathrm{e}} / \mathrm{n}_{\text {tot }}$ & $<0.55$ & $<0.43$ & $0.955-0.985$ \\
$n_{\text {tot }}\left(\mathrm{cm}^{-3}\right)$ & $>0.14$ & $>0.034$ & $0.18-0.29$ \\
$p / k\left(\mathrm{~cm}^{-3} \mathrm{~K}\right)$ & $>1300$ & $>440$ & $2300-5000$ \\
length $(\mathrm{pc})$ & $<1.3$ & $<3.3$ & $0.17-0.45$ \\
\hline
\end{tabular}

\section{Ionization fractions and ionization processes}

From the comparison of the total and the neutral hydrogen column densities derived from $N(\mathrm{~S}$ II $)$ and $N(\mathrm{O}$ I) respectively (see Sect. 4), we estimate the ionization fractions $n_{\mathrm{e}} / n_{\text {tot }}$, listed in Table 4 .

Component 3 is ionized by more than $95 \%$, making its ionization comparable to that of the two main components $(\mathrm{C}$ and $\mathrm{D})$ in the line of sight to $\beta \mathrm{CMa}$ (Jenkins et al. 2000). As for Components $\mathrm{C}$ and $\mathrm{D}$, the main ionizing source for Component 3 is probably the star $\epsilon \mathrm{CMa}$ and its high hydrogen ionization fraction requires that it is located closer to the star, further away from the Sun.

Component 1 (the LIC) can be at most $55 \%$ ionized, its maximum ionization fraction corresponding to the case of no oxygen depletion in the local cloud. However, formally the column density ranges allow the LIC to be neutral.

Since for Component 2 the neutral gas and total gas column density ranges are nearly coincident, Component 2 is most probably neutral although formally its ionization fraction can be as high as $43 \%$ in the case of no oxygen depletion.

In order to compare these numbers to the expected ionization fractions, we have calculated the photoinionization equilibria in the local ISM for varying depths of shielding by neutral $\mathrm{H}$ and $\mathrm{He}$ following the equations described by Sofia \& Jenkins (1998). We consider the effect of Vallerga's (1998) composite stellar radiation field supplemented by Slavin's (1989) calculated flux from the cloud conductive interface. The results are shown in Fig. 7. The calculation is the same as that used to produce Fig. 2 of Jenkins et al. (2000) except for a higher pressure $p / k$, in better agreement with the electron density derived in Sect. 5 for the

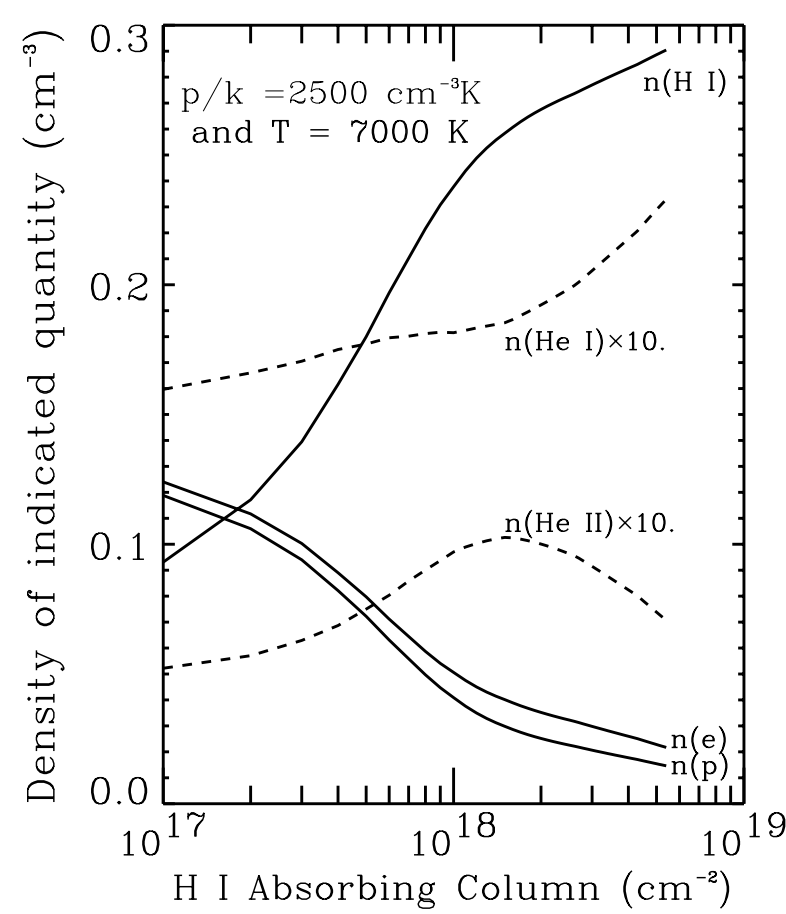

Fig. 7. Predicted densities versus $\mathrm{H}$ I attenuation column density in a local cloud exposed to the local photoionization field (due to hot stars and hot-gas conductive interface)

LIC as well as with the neutral density derived from EUV stellar spectra and inside the heliosphere (Vallerga 1996; Quémarais et al. 1994). We derive the expected ionization fraction in the two local clouds from Fig. 7 and from the estimate of the mean attenuation of the ionizing flux received in the clouds. Since most of the EUV radiation comes from the direction of $\epsilon$ CMa where nearly all the neutral gas is included in one of these two components, the attenuation is derived from their $\mathrm{H}$ I column densities given in Sect. 4.1.

If we assume that Component 2 is located outside of the LIC, further out toward $\epsilon$ CMa, it should be shielded only by its own Hi material, with a mean Hi attenuation of $1 / 2 N(\mathrm{HI})$, in all cases lower than $2.310^{17} \mathrm{~cm}^{-2}$. With this upper limit, Fig. 7 predicts for Component 2 a minimum ionization fraction of 0.44 , which is inconsistent with the slightly lower maximum ionization fraction permitted by the observations. Note that the temperature of 
Component 2 derived in Sect. 5 is higher than that used in Fig. 7, optimized to match the characteristics of the LIC. As the ionization fraction increases with temperature because the recombination rate is a decreasing function of $T$, the predicted ionization fraction for Component 2 is even higher than that which appears in Fig. 7, amplifying further the discrepancy with the value derived from the observations. This inconsistency would be eliminated if Component 2 were shielded by the LIC, which would imply that it is located within the Local Cloud, a configuration already proposed by Gry (1996) for some of the small components detected close to the Sun in several lines of sight.

For the LIC, the mean attenuation due to the cloud itself ranges from $1.510^{17}$ to $2.210^{17} \mathrm{~cm}^{-2}$ depending on the assumed oxygen depletion value. From Fig. 7, this implies an ionization fraction between 0.45 and 0.5 . If we were to add the attenuation effect due to Component 2 (which is maximum if Component 2 is external and shielding the LIC as a whole), the range of possible absorbing column is extended up to 4 or $610^{17} \mathrm{~cm}^{-2}$ and we would get an ionization fraction down to 0.34 or 0.24 . These values are all compatible with the range derived for the LIC from the measured column densities.

Figure 7 also predicts that the ratio $n(\mathrm{He} \mathrm{I}) / n(\mathrm{HI})$ is almost constant over the range of $\mathrm{H}$ I column densities measured toward the white dwarf stars observed by EUVE (around $110^{18} \mathrm{~cm}^{-2}$ ) and is close to the measured ratio of 0.07 (Dupuis et al. 1995). We conclude from the adequate prediction of the hydrogen ionization fraction as well as of the $n(\mathrm{He} \mathrm{I}) / n(\mathrm{HI})$ ratio that the photoionization by the EUV radiation field due to the combination of the hot stars and the cloud conductive interface is a likely representation of the ionization processes in the LIC.

\section{Other physical properties of the clouds}

From the comparison of the ionization fractions and the electron density, we infer the total hydrogen density $n_{\text {tot }}$ (or its lower limit) for each of the three components. These are listed in Table 4 . In fact the numbers are compatible with all three components having a similar total density close to $0.2-0.3 \mathrm{~cm}^{-3}$ but different electron densities depending on their ionization state.

The thermal pressures in the components are also listed in Table 4. To estimate the thermal pressure in the clouds from $p / k=n T$ we sum up over all particles: hydrogen $\left(n_{\text {tot }}=n_{\mathrm{HI}}+n_{\mathrm{e}}\right)$, helium $\left(0.1 n_{\text {tot }}\right)$ and electrons $\left(n_{\mathrm{e}}\right)$. For the LIC, if we adopt the $n_{\mathrm{HI}}$ range of Vallerga (1996), i.e. 0.15 to $0.34 \mathrm{~cm}^{-3}$, with our ranges for $n_{\mathrm{e}}$ and $T$, we derive $p / k=1900$ to $6000 \mathrm{~cm}^{-3} \mathrm{~K}$, in agreement with the thermal pressure found in Component 3, as is expected if they are in equilibrium with a surrounding medium that is common to both of them.

The thicknesses of the components along the line of sight are estimated from the ratio of the total column density $N\left(\mathrm{H}_{\text {tot }}\right)$ derived in Sect. 4.2 to the total density $n_{\text {tot }}$. The lengths of the components (listed in Table 4) are all very small compared to the length of the line of sight: they occupy a total of less than 5 pc. This implies that at least $96 \%$ of the sight-line is empty or filled with more highly ionized gas.

\section{High ionization species and cloud interfaces}

Substantial amounts of Si III are detected in all components. We find that $N(\mathrm{Si}$ III $)=0.5 N(\mathrm{Si}$ II $)$ in Component 1 and more than $0.7 N(\mathrm{SiII})$ in Component 3. While $N$ (Si III) is only about $10 \%$ the value of $N(\mathrm{SiII})$ in Component 2, it is 7.5 times $N$ (Si II) in Component 4.

In principle Si III cannot come from the same region as the other species because charge exchange with even small amounts of neutral hydrogen tends to shift the Si to lower stages of ionization (Jenkins et al. 2000). Nevertheless, it seems clear that Si III arises at velocities that coincide with all components, including even the less ionized Component 2. This makes it is very likely that Si III is located in regions associated with the clouds, perhaps in their outermost layers.

It is interesting to note that Si III is not detected in the spectrum of $\alpha$ CMa (Hébrard et al. 1999) up to a limit of $210^{11} \mathrm{~cm}^{-2}$. This limit corresponds to our detection for Component 2, but it is more than 10 times lower than the column density we derive for the LIC. This discrepancy could be interpreted as Si III coming from a completely unrelated cloud, which coincidently has about the same velocity as the LIC, as proposed by Hébrard et al. (1999).

A difficulty with the above proposal is that this component probably would contaminate other lines, and thus it should influence the column density determinations for other elements. This hypothetical extra component would be likely to have elemental abundance ratios different from the LIC - due in particular to different ionization fractions - and thus create large perturbations in the column density ratios. Yet, if we compare the results for the four species for which we have reliable results for both $\alpha \mathrm{CMa}$ and $\epsilon$ CMa sight-lines (i.e. derived from unsaturated lines): N I, Si II, Fe II and Mg II, the column density ratios between $\epsilon \mathrm{CMa}$ and $\alpha \mathrm{CMa}$ are very similar for all elements: $N(\epsilon \mathrm{CMa}) / N(\alpha \mathrm{CMa})=1.5 \pm 0.2 \mathrm{~cm}^{-2}$. As an example of the kind of differences one can expect between two different components, the column density ratios between Component 1 and Component 3 for the three ionized species Si II, Fe II and Mg II present a dispersion of $60 \%$. Even worse, the N I column density ratio between Component 1 and Component 3 is more than a factor of 10 higher than the ratios for the other elements.

Thus, in view of the small dispersion in the column density ratios between the LIC in the $\epsilon$ CMa sight-line and the LIC in the $\alpha$ CMa sight-line in the various elements, we strongly support the idea that there is only one absorbing component at the LIC velocity in the $\epsilon$ CMa sight-line, and thus that the Si III absorption is related to the LIC component.

The fact that $N(\mathrm{Si}$ III) is at least a factor of 10 lower in the Sirius sight-line than in the $\epsilon$ CMa sight-line suggests 
that the region where the Si III originates is extended and that more than $90 \%$ of it lies beyond Sirius. The possible very small velocity shift between the Si III absorption and the absorption from less ionized species could be explained by the presence of a slight positive velocity gradient toward the outer layer of the cloud.

The detection of SiIII in the LIC has an interesting consequence for the relative location of the two local clouds. Since by definition the LIC is the cloud in which the Sun is embedded, the presence of an extended LIC Si III layer past Sirius implies that the LIC and its extended layer occupy the full line of sight toward Sirius. It follows that Component 2 (the "Blue Component" in the $\alpha$ CMa sight-line) should be embedded in the LIC or at least in its extended Si III layer, corroborating the suggestion made in Sect. 6 .

There is a significant difference between the gas responsible for the SiIII absorption and the gas responsible for the CIV absorption: while the widths of the Si III profiles are compatible with Si III being at the same temperature as the less ionized elements, the profiles for C IV are clearly broader, implying temperatures of the order of 100000 to $200000 \mathrm{~K}$. This favours the existence of collisional ionization due to a high temperature. Indeed, our C IV column densities and our Si IV upper limits are compatible with the outcome from Slavin's (1989) model for the conduction layer between the Local Cloud and the hot gas that is supposed to fill the Local Bubble. He calculated that $N(\mathrm{C} \mathrm{IV})=2.710^{12} \mathrm{~cm}^{-2}$ and $N(\mathrm{Si} \mathrm{IV})=110^{11} \mathrm{~cm}^{-2}$ through an interface of this sort, assuming minimal inhibiting effects from magnetic fields. His prediction for $N$ (Si III) of $510^{10} \mathrm{~cm}^{-2}$ is far below our observed column density.

\section{Summary}

We have analysed the interstellar absorption lines in the high spectral resolution $(R \sim 100000)$ UV spectrum of $\epsilon$ CMa $(130 \mathrm{pc})$. We derive column densities for 11 different elements in the three main clouds and for a few elements with the strongest lines in two additional very weak components.

Two of the main components (Components 1 and 2) are identified with the two components detected in the much shorter line of sight toward $\alpha \mathrm{CMa}$ (Sirius) which is not far from $\epsilon$ CMa in the sky. One of them (Component 1) is the Local Interstellar Cloud (LIC) in which the Sun is embedded. For the four elements for which reliable measurements exist for the two lines of sight (i.e. performed with unsaturated lines) we find a constant ratio of 1.5 between the column densities of the LIC toward $\epsilon$ CMa and the column densities of the LIC toward $\alpha$ CMa.

We derive the neutral hydrogen column density from our measurement of $\mathrm{O} I$ which is a good tracer of $\mathrm{HI}$. Depending on the oxygen abundance we adopt, we find a neutral gas column density for the whole line of sight between $6.0 \pm 1.210^{17} \mathrm{~cm}^{-2}$ if we consider that the local gas is not depleted (adopting the B stars abundance) and $9.0 \pm 2.010^{17} \mathrm{~cm}^{-2}$ if we adopt the mean ISM oxygen abundance of Meyer et al. (1998). With the same two alternatives, we derive for the LIC a neutral gas column density between $3.0_{-0.4}^{+1.1} 10^{17} \mathrm{~cm}^{-2}$ and $4.4_{-0.6}^{+1.6} 10^{17} \mathrm{~cm}^{-2}$.

We estimate the temperatures and electron densities in the three main components by combining the information of the two ratios $N\left(\mathrm{CII}^{*}\right) / N(\mathrm{C}$ II $)$ and $N(\mathrm{Mg} \mathrm{II}) / N(\mathrm{Mg} \mathrm{I})$. In particular for the LIC we find $n_{\mathrm{e}}=0.12 \pm 0.05 \mathrm{~cm}^{-3}$ and $T=7000 \pm 1200 \mathrm{~K}$, both in agreement with previous determinations having similar error bars.

We compare the neutral gas column densities with the total (neutral and ionized) gas column densities derived from the S II measurements, to conclude that Component 3 is ionized by more than $95 \%$, that Component 2 is probably neutral but could be as much as $43 \%$ ionized and that the LIC can be at most $55 \%$ ionized. We conclude from these numbers that Component 3 must be located further away on the line of sight and is thus almost fully ionized by $\epsilon$ CMa and that the ionization fraction in the LIC is compatible with the gas being ionized by the local EUV radiation fields from the hot stars and the cloud interface with hot gas. In contrast, it is hard to explain the low state of ionization of Component 2 unless it is included within the LIC which shields it from the ionizing radiation.

We detect high ionization species. Si III is detected in all clouds but more significantly in the LIC and Component 3, and is probably located in extended layers in the outer regions of the clouds. C IV is also detected in the LIC and Component 3, but with small velocity offsets from the lower ionization species. The derived amount of highly ionized gas and the derived high temperature are consistent with the predictions of Slavin (1989) for a conductive interface between the Local Cloud and the surrounding hot gas from the Local Bubble.

Acknowledgements. The GHRS data reduction and a preliminary spectral analysis have been performed in collaboration with Olivier Dupin as part of his Ph.D. Thesis, presented in April 1998. CG is very grateful to Martin Lemoine for his absorption line fitting software "Owens" and his helpful advices. EBJ was supported by NASA grant NAG5-616 to Princeton University.

\section{References}

Allan, R. J., Clegg, R. E. S., Dickinson, A. S., \& Flower, D. R. 1988, MNRAS, 235, 1245

Anders, E., \& Grevesse, N. 1989, Geochim. Cosmochim. Acta, 53, 197

Cassinelli, J. P., Cohen, D. P., MacFarlane, J. J., et al. 1995, ApJ, 438, 932

Dupin, O., \& Gry, C. 1998, A\&A, 335, 661

Dupuis, J., Vennes, S., Bowyer, S., \& Pradhan, A., Thejll, P. 1995, ApJ, 455, 574

Field, G. B., \& Steigman, G. 1971, ApJ, 166, 59

Fitzpatrick, E. L., \& Spitzer, L. 1997, ApJ, 475, 623

Frisch, P. C., \& Slavin, J. D. 1996, Space Sci. Rev., 78, 223

Gry, C., Lemonon, L., Vidal-Madjar, A., Lemoine, M., \& Ferlet, R. 1995, A\&A, 302, 497 
Gry, C. 1996, Space Sci. Rev., 78, 239

Hayes, M. A., \& Nussbaumer, H. 1984, A\&A, 134, 193

Hébrard, G., Mallouris, C., Ferlet, R., et al. 1999, A\&A, 350, 643

Hoffleit, D., \& Jaschek, C. 1982, The Bright Star Catalogue, 4th ed. (New Haven: Yale U. Obs.)

Holberg, J. B., Bruhweiler, F. C., Barstow, M. A., \& Dobbie, P. D. 1999, ApJ, 517, 841

Hurwitz, M., Bowyer, S., Bristol, R., et al. 1998, ApJ, 500, L1

Jenkins, E. B., Reale, M. A., Zucchino, P. M., \& Sofia, U. J. 1996, Astr. Space Sci., 239, 315

Jenkins, E. B., Gry, C., \& Dupin, O. 2000, A\&A, 354, 253

Jenkins, E. B., Oegerle, W. R., Gry, C., et al. 2000, ApJ, 538, L81

Lallement, R., \& Bertin, P. 1992, A\&A, 266, 479

Lallement, R., Bertin, P., Ferlet, R., Vidal-Madjar, A., \& Bertaux, J. L. 1994, A\&A, 286, 898

Lallement, R., \& Ferlet, R. 1997, A\&A, 324, 1105

Linsky, J. L., Diplas, A., Wood, B. E., Brown, A., Ayres, T. R., \& Savage, B. D. 1995, ApJ, 451, 335

Lyu, C. H., \& Bruhweiler, F. C. 1996, ApJ, 459, 216

Meyer, D. M., Cardelli, J. A., \& Sofia, U. J. 1997, ApJ, 490, L103

Meyer, D. M., Jura, M., \& Cardelli, J. A. 1998, ApJ, 493, 222

Morton, D. C. 1991, ApJS, 77, 119
Nussbaumer, H., Storey, P. J. 1981, A\&A, 96, 91

Nussbaumer, H., Storey, P. J. 1986, A\&AS, 64, 545

Perryman, M. A. C., Lindegren, L., Kovalevsky, J., et al. 1997, A\&A, 323, L49

Quémarais, E., Bertaux, J. L., Sandel, B., Lallement, R. 1994, A\&A, 290, 941

Reynolds, R. J. 1986, AJ, 92, 653

Savage, B. D., \& Sembach, K. R. 1996, ARA\&A, 34, 279

Sembach, K. R., Savage, B. D., \& Jenkins, E. B. 1994, ApJ, 421,585

Shull, J. M., \& van Steenberg, M. 1982, ApJS, 48, 95

Slavin, J. 1989, ApJ, 346, 718

Slavin, J. D., \& Frisch, P. C. 1998, in The Local Bubble and Beyond, ed. Breitschwerdt, Freyberg, \& Trumper (Berlin: Springer), 305

Sofia, U. J., \& Jenkins, E. B. 1998, ApJ, 499, 951

Soderblom, D. R., Gonnella, A., Hulbert, S. J., et al. 1995, GHRS Instrument Handbook version 6, Space Telescope Science Institute

Vallerga, J. V. 1996, Space Sci. Rev., 78, 277

Vallerga, J. V. 1998, ApJ, 497, 921

Vallerga, J. V., \& Welsh, B. Y. 1995, ApJ, 444, 702

Vallerga, J. V., Vedder, P. W., \& Welsh, B. Y. 1993, ApJ, 414, L65

Wood, B. E., \& Linsky, J. L. 1997, ApJ, 474, L39 